\title{
Establishing safe transfers between acute medical and psychiatric inpatient facilities
}

\author{
Author: Jonathan Mamo
}

\section{Aims}

To improve the communication between two NHS trusts at the pre-transfer phase thereby ensuring safe transfer of medically fit individuals to a psychiatric hospital.

\section{Methods}

A questionnaire was submitted to all doctors working within the psychiatric department to elicit what issues were experienced in patient transfers and what markers would be considered essential in any transfer checklist. The results of the questionnaire were followed by the introduction of a pre-transfer medical checklist. The designed checklist and guideline required doctor-to-doctor discussion across the two sites to ensure confirmation of the agreed minimum medical criteria for safe transfer to a psychiatric bed. A simple-to-follow one-page form was created to ensure appropriate documentation of requested status.

\section{Results}

Initial responses to the questionnaire reported a pre-cycle unsafe transfer of $71 \%$ with the main issues being missing discharge letters, missing drug list and discharge letters with no valuable information. All respondents to a departmental questionnaire indicated that a transfer checklist would be of benefit. We conducted a 'plan, do, study, act' cycle within a quality improvement model. The checklist went live once stakeholders had an opportunity to contribute to its creation. Following a trial period of its use, we collected data from all adult and old patient transfers from regional acute hospitals to psychiatric units within Portsmouth. We established the level of checklist completion and acquired feedback from involved parties. A conservative estimate shows a cost saving of $£ 40,000$ on staff time saved. This does not take into consideration transport costs, drug costs and error costs. The savings of a small change have already been shown to be of great benefit and further analysis of cost savings will be assessed over time.

\section{Conclusion}

The transfer of patients from acute hospitals to psychiatric inpatient facilities requires clear communication and careful

Author: Royal College of Physicians chief registrar, Solent NHS Trust, UK coordination of care in order to maximise patient safety. Anecdotal evidence suggests handover information at transfer is variable. This has sometimes resulted in patient safety concerns, and it is felt that there is a need for an established standard. In doing so, we hope to safeguard the safety of our patients and instil confidence in health care professionals involved in their transfer.

\section{Conflict of interest statement}

No conflict of interest. 